\title{
Tabaquismo activo y cáncer pulmonar: Determinación de fracciones atribuibles por sexo
}

\author{
Marcia Erazo $\mathrm{B}^{1,2}$, Hugo Amigo $\mathrm{C}^{2}$, Manuel 0 yarzún $\mathrm{G}^{3}$, \\ Armando Peruga U 4 .

\section{Smoking and lung cancer: Attributable risks according to gender}

Background: The association between lung cancer and smoking is well documented. However there is less information about the estimation of its attributable fraction and population burden. Aim: To estimate the attributable risk and population attributable risk of smoking among lung cancer patients attended in Public Health Services at Santiago. Material and methods: A case control study matched by age was carried out. Crude and adjusted attributable and population attributable risks were estimated, controlling for potential confounders and interaction variables. Results: Mean age for cases was 63 years for women and 67 years for men. Lung cancer patients had a higher smoking prevalence than controls (64.5\% and 39.7\% respectively among women; 95.8 and 67.1 respectively among men $\mathrm{p}<0.01$ ). Heavy smoker proportion was 4 times higher among patients that smoked 5 to 10 years more (women and men respectively, $\mathrm{p}<0.01$ ) and 3 times more cigarettes per day ( $p$ $<0.01$ ). Attributable risk for women was $64.4 \%$ and $90.4 \%$ for men. Population attributable fraction was $41.9 \%$ and $86.3 \%$ for women and men, respectively. Projecting these estimates to the Chilean population, approximately 1975 new cases per year of lung cancer caused by smoking will be diagnosed. Conclusions: Attributable risks of smoking for lung cancer are high and significant, even when they are adjusted by confounding variables (Rev Méd Chile 2008; 136: 1272-80).

(Key words: Lung neoplasms; Smoking; Tobacco smoke pollution)

Recibido el 4 de enero, 2008. Aceptado el 10 de julio, 2008.

Trabajo financiado por la Organización Panamericana de la Salud Grant \# 560112 y Beca de Post Grado de la Comisión Nacional para la Investigación Científica y Tecnológica (CONICYT).

${ }^{1}$ Programa de Doctorado en Salud Pública, Universidad de Chile. ${ }^{2}$ Facultad de Medicina, Universidad de Chile. Santiago, Chile. ${ }^{3}$ Laboratorio de Investigaciones Respiratorias, Instituto de Ciencias Biomédicas, Facultad de Medicina, Universidad de Chile. ${ }^{4}$ Tobacco-free initiative, National Capacity Building Unit Coordinator, World Health Organization. Ginebra, Suiza.

Correspondencia a: Dra. Marcia Erazo B. Independencia 1027, Santiago, Chile. Fono: 9786213. Fax: 7355581.

E mail: merazo@med.uchile.cl 
$\mathrm{E}_{\mathrm{te}}^{\mathrm{n}}$ América Latina, el uso de tabaco es frecuente, con una alta prevalencia en hombres y una mortalidad relativamente baja ${ }^{1}$. En Chile, la prevalencia es superior a $40 \%$ en la población general, observándose un incremento en mujeres y una disminución en la edad de inicio de consumo ${ }^{2}$.

El tabaquismo es la principal causa de discapacidad y muerte prematura prevenible, y entre las patologías derivadas de su consumo está el cáncer pulmonar ${ }^{3}$. El carácter etiológico ha sido ampliamente estudiado y publicado en diversos estudios epidemiológicos ${ }^{3-5}$, estableciéndose su asociación causal en la década 1950-59 con los estudios de Doll y Hill 6 .

Como ya ha sido señalado, la asociación entre tabaquismo y cáncer pulmonar ha sido documentada previamente, sin embargo, el cálculo de su fracción atribuible y cuantificación poblacional ha sido menos estudiada. Esta cuantificación es importante debido a que es posible estimar qué fracción de la población con cáncer pulmonar se evitaría si la exposición a tabaquismo no existiera, por lo tanto apoya de manera directa la implementación de políticas tendientes a disminuir su consumo. El objetivo de este estudio, que se enmarca en un proyecto multicéntrico de cuatro países de América Latina, coordinado por la Organización Panamericana de la Salud (OPS), es estimar el impacto del consumo de tabaco sobre la ocurrencia de cáncer del pulmón en la población de Santiago, Chile.

\section{MATERIAL Y MÉTODO}

Estudio de casos y controles pareado por edad y sexo. Este trabajo se enmarcó en un estudio multicéntrico de cuatro países de América Latina (Brasil, Colombia, México y Chile) que estudió los costos de atención en tres patologías atribuibles al tabaquismo (infarto agudo al miocardio, cáncer pulmonar y enfermedad obstructiva crónica) y fue coordinado por la Organización Panamericana de la Salud (OPS). En este estudio multicéntrico hubo dos fases: la epidemiológica y la económica, que siguió técnicas estandarizadas en la recolección de información y análisis de datos; y en este artículo se comunican los hallazgos de la fase epidemiológica del estudio realizado en Chile.
Como criterio de elegibilidad se consideró que casos y controles debieron vivir en Santiago a lo menos durante los últimos 5 años previos a la entrevista. Tanto casos como controles fueron reclutados entre marzo de 2001 y enero de 2006.

Caso fue aquel individuo que presentó diagnóstico clínico de cáncer pulmonar primario hasta dos años después de hecho el diagnóstico, con una biopsia realizada en el hospital donde se atendía e informada por el anatomopatólogo del servicio. El diagnóstico histopatológico fue corroborado por un segundo patólogo.

Para seleccionar a los casos, primero se identificaron los hospitales que atendían a pacientes con cáncer de pulmón y en cada hospital se identificaron los servicios asociados a su diagnóstico y tratamiento, construyéndose posteriormente un listado de los pacientes por centro asistencial. Se verificó si alguno de ellos se encontraba hospitalizado, se les explicaron los objetivos del estudio e invitó a participar en la investigación y una vez que aceptó, fue entrevistado. Los casos provinieron de los siguientes hospitales de Santiago: Instituto Nacional del Tórax, Instituto Nacional del Cáncer, Hospital Barros Luco-Trudeau, Hospital Sótero del Río y Hospital Clínico de la Universidad Católica.

Para cada caso se seleccionó en forma aleatoria dos pacientes de igual sexo y edad ( \pm 3 años) que el caso. Los controles no tuvieron antecedentes de cáncer de ningún tipo, tuberculosis ni infarto agudo del miocardio, información que fue corroborada con la ficha clínica. La decisión de tomar dos controles por caso fue con el fin de aumentar el poder del estudio.

Los controles fueron reclutados de los servicios ambulatorios de oftalmología, otorrinolaringología, traumatología, ginecología y cirugía del complejo asistencial donde se obtuvo el caso y fueron elegidos del listado de personas que estaban citados para atención profesional en los servicios antes mencionados, hasta un mes después del reclutamiento del caso. Del listado de pacientes, se procedió a sortear a los que se invitó a participar como controles en el estudio y si alguno de ellos no deseaba participar, se procedió a sortear otro paciente del listado.

El tamaño muestral fue de 138 casos y 276 controles mujeres y 72 casos y 144 controles 
hombres. Para estimar el tamaño de muestra se especificó un nivel de significancia de 0,05 y una potencia (1-b) del test de $80 \%$ y test de un lado. Para estos cálculos, se consideró 20\% de pares discordantes y un odds ratio (OR) esperado de 2. El cálculo fue realizado según la fórmula de Machin y Lachin 7,8 .

La información fue recolectada en cuestionarios especialmente diseñados por especialistas de los cuatro países involucrados en el estudio multicéntrico y de la Organización Panamericana de la Salud, el que consideraba una detallada información acerca de la historia de tabaquismo de la persona (edad de inicio, número de cigarrillos y frecuencia de consumo (diaria, semanal, anual), profundidad de la inhalación y año de cesación), así como potenciales fuentes de contaminación laboral e intradomiciliaria (tabaquismo ambiental, exposición a carbón, leña, parafina, asbesto y radón). La entrevista fue realizada por enfermeras capacitadas y supervisadas de manera permanente, previa firma de un acta de consentimiento informado. El proyecto fue aprobado por el Comité de Ética de la Investigación en Seres Humanos, Facultad de Medicina, Universidad de Chile.

El procesamiento de la información se realizó en el programa EPI Data versión 2.19, efectuándose doble digitación a fin de verificar inconsistencias.

El análisis estadístico se realizó en diferentes etapas:

Primero: una fase exploratoria de los datos, identificando distribución, valores atípicos y estimando medidas de tendencia central y dispersión. Luego las variables fueron categorizadas (ej: intensidad de fumador) y se crearon indicadores (ej: paquete año, fumador pasivo, exposición laboral o domiciliaria a otros contaminantes).

Posteriormente se estimaron los OR en forma cruda y ajustada entre las distintas exposiciones de tabaco y cáncer pulmonar a través de modelos de regresión logística condicional, controlando por posibles efectos de confusión, considerando significativos valores de $p<0,05$. A partir de los OR se calculó la fracción atribuible y finalmente la fracción atribuible poblacional.
La estimación de la fracción atribuible (FA) se realizó de acuerdo a la siguiente fórmula ${ }^{10}$ :

$\mathrm{FA}=\frac{(\mathrm{OR}-1)}{\mathrm{OR}}$

y para la fracción atribuible poblacional (FAP) ${ }^{11}$

$F A P=\frac{p_{e} \times(O R-1)}{p_{e} \times(O R-1)+1}$

$\mathrm{p}_{\mathrm{e}}=$ Proporción de expuestos entre los controles

La transformación de las variables se realizó utilizando SPSS versión $12.0^{12} \mathrm{y}$ el análisis en el programa Stata versión $8.0^{13}$.

\section{Resultados}

La diferencia en edad entre casos hombres y mujeres fue de 4 años. En relación a la escolaridad, las diferencias entre casos y controles fueron de un año en hombres y en las mujeres, tres (Tabla 1). Tanto hombres como mujeres presentaban una alta exposición a contaminantes en el domicilio superando $90 \%$, en cambio en el lugar de trabajo, ésta era baja. La historia familiar de cáncer pulmonar fue más prevalente en mujeres que en hombres (Tabla 1).

En relación al tipo histológico de neoplasia pulmonar, en los hombres predominó el carcinoma epidermoide y en las mujeres el adenocarcinoma. La información sobre los valores de riesgo según tipo histopatológico y su impacto poblacional será motivo de otra publicación.

Los hombres con cáncer de pulmón, al ser comparados con las mujeres, presentaban mayor prevalencia de tabaquismo en la vida (30\% más), la proporción de grandes fumadores, definidos como quienes fuman más de 20 paquetes-año*, fue 2 veces mayor y consumieron en promedio 5 cigarrillos diarios más. Sin embargo, siguieron siendo fumadores activos en similar proporción y fumaron una cantidad similar de años. Al ser comparados con sus controles, en ambos sexos los casos presentaron mayor prevalencia de vida de tabaquismo, menor proporción de fumadores activos,

*paquetes-año $=\left(\underline{n} n^{0}\right.$ de cigarrillos/día $)$ x número de años fumando 
Tabla 1. Características sociodemográficas y de exposición a tabaco y a otros contaminantes de los casos y controles del estudio según sexo

\begin{tabular}{|lcccc|}
\hline & \multicolumn{2}{c}{ Mujeres } & \multicolumn{2}{c|}{ Hombres } \\
& Casos & Controles & Casos & Controles \\
& $(\mathrm{n}=138)$ & $(\mathrm{n}=276)$ & $(\mathrm{n}=72)$ & $(\mathrm{n}=144)$ \\
& & & & \\
\hline Socio-demográfico & & & & \\
$\quad$ Edad (años) & $63(56-71)$ & $63(55-72)$ & $67(57-72)$ & $66(58-72)$ \\
Educación (años) & $9(6-13)$ & $6(4-10)$ & $9(6-12)$ & $8(4-12)$ \\
Otras exposiciones* & & & & \\
Trabajo (\%) & 7,9 & 18,3 & 20,5 & 46,6 \\
Hogar (\%) & 89,9 & 95,7 & 90,4 & 97,3 \\
Historia familiar de cáncer pulmonar (\%) & 18,7 & 5,4 & 9,6 & 2,1 \\
Tabaquismo activo & & & & \\
Haber fumado en la vida (\%) & 64,5 & 39,7 & 95,8 & 67,1 \\
- Actual (\%) & 13,6 & 34,6 & 11,6 & 27,6 \\
- Exfumador (\%) & 86,4 & 65,4 & 88,4 & 72,4 \\
Paquete-año: & & & & \\
- No-fumadores (\%) $\quad 35,5$ & 60,2 & 4,2 & 32,9 \\
- $\quad$ 0,1 a 19,99 (\%) & 27,5 & 31,5 & 20,8 & 45,2 \\
- $\quad 20$ (\%) & 37,0 & 8,2 & 75,0 & 21,9 \\
Edad de inicio (años) & $18(14-20)$ & $20(15-25)$ & $17(14-18)$ & $17(15-20)$ \\
Duración (años) & $35(26-47)$ & $25(15-39)$ & $40(30-49)$ & $35(20-44)$ \\
Cigarrillos por día & $15(5-10)$ & $4(2-10)$ & $20(10-30)$ & $6(2-20)$ \\
Tabaquismo Involuntario & & & & \\
General: & & & & \\
- Vivir con fumador (\%) & 76,1 & 69,2 & 62,5 & 59,6 \\
- Trabajar con fumador (\%) & 44,2 & 24,0 & 61,1 & 32,2 \\
Entre no fumadores: & $(n=51)$ & $(n=171)$ & $(\mathrm{n}=4)$ & $(\mathrm{n}=48)$ \\
- Vivir con fumador (\%) & 66,7 & 63,7 & 75,0 & 56,3 \\
- Trabajar con fumador (\%) & 33,3 & 19,9 & 50,0 & 25,0 \\
\hline
\end{tabular}

Valores representan mediana (dispersión intercuartílica) o porcentaje.

*En el trabajo se consideró exposición a asbesto, gas radón, plomo y arsénico. En el hogar las exposiciones fueron: leña, kerosene y carbón.

el número de cigarrillos diarios fue 3 veces mayor, aunque iniciaron a edades similares el consumo de cigarrillo. El tipo de cigarrillo más consumido por hombres y mujeres era el con filtro; los casos lo utilizaron $20 \%$ más que sus controles. En relación a la profundidad de la inhalación, la aspiración leve a moderada fue la más prevalente en ambos sexos. La exposición a tabaquismo ambiental en toda la muestra fue alta, superando $50 \%$ en el hogar, resaltando que las mujeres estaban más expuestas que los hombres, situación que era inversa en el lugar de trabajo (Tabla 1).
Cuando se analiza el riesgo de cáncer pulmonar por sexo, aquellos que habían fumado más de cien cigarrillos en su vida (límite entre fumador y no fumador), tenían una diferencia de cinco veces en la fuerza de asociación, siendo mayor en hombres. En los que fumaron menos de 20 paquetes-año, el riesgo de cáncer pulmonar fue el doble en hombres que en mujeres, aunque no son estadísticamente significativos en ninguno de los dos casos. En los grandes fumadores (más de 20 paquetes año), el riesgo para hombres fue más de seis veces mayor que para las mujeres. Al ajustar por variables 
de confusión potenciales, el riesgo en los hombres se hizo casi 60 veces mayor que entre los no fumadores, situación opuesta a lo observado en las mujeres que apenas se modificó después del ajuste, y por lo que la diferencia del riesgo de adquirir cáncer de pulmón fue de casi diez veces mayor para los hombres. Las mujeres presentaron una relación dosis respuesta en sus riesgos cuando se considera el uso de filtro en el cigarrillo, siendo los valores significativos en ambas categorías. Cuando se analiza la profundidad de la inhalación, el riesgo de adquirir cáncer pulmonar no fue diferente en los dos tipos de inhaladores, ni en hombres ni en mujeres (Tabla 2).

En los individuos que habían fumado al menos 100 cigarrillos en su vida, el valor de la FA calculada en las mujeres fue poco mayor de $60 \%$. En los hombres, este valor fue levemente mayor a 90\% (Tabla 3). Cuando se estiman las fracciones atribuibles poblacionales, se observó que ésta fue levemente mayor a $40 \%$ en mujeres, valor que se duplicó para los hombres (Tabla 3).

El número de habitantes del país mayor de 20 años que cada año se diagnosticarían con cáncer pulmonar atribuible al consumo de al menos 100 cigarrillos en la vida llegaría a casi 2.000, resaltando que la cantidad de hombres es tres veces mayor que las mujeres. Hipotetizando, que a partir de la implementación de la ley anti-tabaco en el país se pudiera disminuir en $5 \%$ su prevalencia de consumo, se evitarían 49 nuevos pacientes con cáncer pulmonar por año, siendo las mujeres las que se diagnosticarían menos que los hombres (29 vs 20) (Tabla 4).

\section{DisCUSIÓN}

En este estudio se comprueba que el tabaquismo es un factor fuertemente asociado a cáncer pulmonar, con una diferenciación por sexo en que los hombres presentan valores superiores a las mujeres a un mismo nivel de exposición de tabaquismo, diferencia que no es explicada por la profundidad de la inhalación ni por el uso de filtro, ya que ambos patrones son muy similares entre mujeres y hombres.

$\mathrm{Si}$ bien los altos valores de asociación con tabaquismo han sido ampliamente reportados en la literatura internacional, en relación al comporta-

\section{Tabla 2. 0 dds ratios (O R) e intervalo de confianza de $95 \%(95 \% \mathrm{CI})$ para el $O \mathrm{R}$, según sexo de acuerdo a distintos grados de exposición a tabaquismo}

\begin{tabular}{|lcccc|}
\hline Exposición a tabaquismo & \multicolumn{2}{c}{ Mujeres } & $\begin{array}{c}\text { Hombres } \\
\end{array}$ \\
& OR crudo $(95 \%$ CI) & OR ajustado* $(95 \%$ CI) & OR crudo $(95 \%$ CI) & OR ajustado* $(95 \%$ CI) \\
\hline $\begin{array}{l}\text { Cantidad fumada } \\
\text { No-fumadores }\end{array}$ & 1,00 (referencia) & 1,00 (referencia) & 1,00 (referencia) & 1,00 (referencia) \\
Haber fumado en la vida & $2,81(1,80-4,38)$ & $2,86(1,83-4,48)$ & $10,45(3,14-34,76)$ & $10,61(3,09-36,40)$ \\
0,1 a 19,99 paq-año & $1,49(0,89-2,51)$ & $1,39(0,77-2,50)$ & $2,65(0,66-10,59)$ & $2,83(0,62-12,92)$ \\
$\geq 20$ paq-año & $7,49(3,97-14,15)$ & $6,24(2,99-13,02)$ & $44,71(9,06-220,70)$ & $57,17(8,46-386,04)$ \\
Tipo cigarillo & & & & \\
No-fumadores & 1,00 (referencia) & 1,00 (referencia) & 1,00 (referencia) & 1,00 (referencia) \\
Con filtro & $1,83(1,20-2,81)$ & $1,83(1,20-2,81)$ & $1,69(0,81-3,50)$ & $1,69(0,81-3,50)$ \\
Sin filtro & $3,94(1,09-14,32)$ & $3,94(1,09-14,32)$ & $2,58(0,86-7,73)$ & $2,58(0,86-7,73)$ \\
Inhalación & & & & \\
No-fumadores & 1,00 (referencia) & $1,00($ referencia) & 1,00 (referencia) & 1,00 (referencia) \\
Leve-moderada & $2,11(1,28-3,46)$ & $2,11(1,28-3,46)$ & $1,92(0,86-4,27)$ & $1,92(0,86-4,27)$ \\
Profunda & $1,70(1,01-2,90)$ & $1,70(1,01-2,90)$ & $2,02(0,90-4,55)$ & $2,02(0,90-4,55)$ \\
\hline
\end{tabular}

*Ajustado por educación, tabaquismo pasivo, exposición a otros contaminantes en el hogar y trabajo y antecedente familiar de cáncer pulmonar. 
Tabla 3. Fracción atribuible (FA), fracción atribuible poblacional (FAP) e intervalo de confianza del $95 \%$ para cada estimador $(95 \% \mathrm{CI})$

\begin{tabular}{|lccccc|}
\hline & Estimador & \multicolumn{3}{c}{ Mujeres } & \multicolumn{2}{c|}{ Hombres } \\
& & $(95 \% \mathrm{CI})$ & $\begin{array}{c}\text { Ajustado* } \\
(95 \% \mathrm{CI})\end{array}$ & $\begin{array}{c}\text { Crudo } \\
(95 \% \mathrm{CI})\end{array}$ & $\begin{array}{c}\text { Ajustado* } \\
(95 \% \mathrm{CI})\end{array}$ \\
\hline $\begin{array}{l}\text { Haber fumado } \\
\text { en la vida }\end{array}$ & FA & $64,41(63,98-64,83)$ & $65,03(64,61-65,45)$ & $90,43(89,22-91,63)$ & $90,57(89,36-91,78)$ \\
& FAP & $41,99(41,85-42,14)$ & $42,66(42,51-42,80)$ & $86,36(86,21-86,50)$ & $86,55(86,40-86,70)$ \\
\hline
\end{tabular}

*Ajustado por educación, tabaquismo pasivo, exposición a otros contaminantes en el hogar y trabajo y antecedente familiar de cáncer pulmonar

\section{Tabla 4. Proyección de población con cáncer de pulmón mayor de 20 años atribuible al tabaco en $\mathrm{C}$ hile. Considerando como exposición: fumar al menos 100 cigarrillos durante su vida}

\begin{tabular}{|c|c|c|c|c|c|}
\hline Población & Población total ${ }^{\dagger}$ & $\begin{array}{c}\text { Incidencia de } \\
\text { cáncer de pulmón* }\end{array}$ & $\begin{array}{l}\text { Población con } \\
\text { cáncer de pulmón }\end{array}$ & $\begin{array}{c}\text { Fracción atribuible } \\
\text { poblacional }\end{array}$ & $\begin{array}{c}\text { Población con } \\
\text { cáncer de pulmón } \\
\text { atribuible al tabaquismo }\end{array}$ \\
\hline \multicolumn{6}{|c|}{ Nacional } \\
\hline Mujeres & 7.668 .740 & 9,4 & 721 & 0,42 & 303 \\
\hline Hombres & 7.447.695 & 26,1 & 1.944 & 0,86 & 1.672 \\
\hline Total & 15.116 .435 & & 2.665 & & 1.975 \\
\hline \multicolumn{6}{|c|}{ Disminución de $5 \%$ en la prevalencia de tabaquismo a nivel nacional } \\
\hline Mujeres & 7.668 .740 & 9,4 & 721 & 0,38 & 274 \\
\hline Hombres & 7.447 .695 & 26,1 & 1.944 & 0,85 & 1.652 \\
\hline Total & 15.116 .435 & & 2.665 & & 1.926 \\
\hline
\end{tabular}

${ }^{\dagger}$ Censo 2002.

*Por 100 mil habitantes. Globocan 2002.

miento por sexo existen publicaciones contradictorias. En los estudios caso-control ${ }^{14-25}$, se reporta que las mujeres presentan valores más altos que los hombres a un mismo nivel de exposición a tabaquismo, situación opuesta a lo encontrado en nuestra investigación.

En esta investigación, las mujeres consideradas como grandes fumadoras tenían un riesgo 7 veces mayor que las no fumadoras de contraer cáncer pulmonar, cifra que concuerda con las reportadas por otros estudios caso-control cuyos valores de odds ratio oscilan entre 0,8 y 81,914-25. En los hombres, nuestro estudio encuentra cifras cerca- nas a 50 para los grandes fumadores, que es mayor a lo informado por otros autores ${ }^{14-25}$, en que las cifras no superan 28, a excepción de un estudio $^{16}$ que señala un riesgo de 113 para los fumadores de más de 61 paquetes-año, aunque con un intervalo de confianza muy amplio.

En relación a la profundidad de la inhalación, también se han publicado estudios caso-control que reportan información por sexo, con una definición de exposición que en ocasiones corresponde a inspiración profunda 0 no y en otras oportunidades hacen más de dos categorías ${ }^{5,26-34}$. En estos estudios, se ha comunicado que aquellos 
pacientes que inhalan presentan mayor riesgo de tener cáncer pulmonar que los que no lo hacen, con valores que van entre 0,9 y 15,4 , no habiendo una diferencia entre hombres y mujeres.

Existe controversia acerca de si la mujer es más susceptible que el hombre a los efectos carcinogénicos del tabaco. En los estudios casocontrol, las mujeres presentan valores de odds ratio mayores que los hombres a un mismo nivel de exposición ${ }^{35}$, en cambio en los de cohorte, los riesgos para las mujeres son menores que para los hombres $^{35}$. Entre las explicaciones que se han dado sobre esta discrepancia destacan: a) un posible sesgo por subreporte de exposición al tabaquismo activo y b) un efecto confundente del tabaquismo pasivo que podría ser mayor en los hombres 35 .

En esta investigación, las mujeres presentaron valores de riesgo menor que los hombres, siendo consistentes con los estudios de cohorte, tal vez debido a que se realizó una identificación exhaustiva de la exposición al tabaquismo en cada individuo. Cabe destacar que estos valores de riesgo de cáncer pulmonar representan el consumo de tabaco de una población que inició el hábito hace aproximadamente 50 años, en que las mujeres presentaban una menor prevalencia $\mathrm{y}$ cantidad de cigarrillos fumados que en los hombres, por lo que resultaría interesante poder evaluar la tendencia de los riesgos en las próximas cohortes de pacientes con cáncer pulmonar, especialmente mujeres, ya que ellas han aumentado la prevalencia de consumo, disminuido la edad de inicio y aumentado la cantidad de cigarrillos fumados ${ }^{36}$.

Independientemente de los estudios epidemiológicos, se ha señalado que el cáncer pulmonar pareciera una enfermedad distinta para mujeres. Entre las razones se encuentran una mayor susceptibilidad genética al estar más predispuestas a aberraciones metabólicas producto del tabaquismo, como mayores mutaciones específicas en los genes p53, CYP1A1, GSTM1 y K-Ras, y mayor cantidad de aductos de ADN a menor nivel de exposición de tabaquismo. Así mismo, presentarían una menor capacidad para reparar los daños en el ADN, independientemente del nivel de tabaquismo ${ }^{35,37}$. También se ha planteado que las hormonas cumplinían un rol en la carcinogénesis al haberse identificado receptores de estrógeno $\alpha$ en tumores de pulmón. En el caso de los receptores de estrógeno $ß$, se expresan tanto en el pulmón sano y en el con tumor causando una proliferación celular en cánceres de células no pequeñas ${ }^{35}$.

Entre las limitaciones de este estudio se podría considerar el sesgo (de Berkson) en la obtención de los casos y de los controles. Se piensa que existe este tipo de sesgo en los casos, especialmente en los hombres, ya que cerca de $100 \%$ era fumador y consumidor de grandes cantidades diarias de cigarrillos, prácticamente no dejando población de referencia, lo que implicó por un lado que los valores de riesgo fueran muy elevados para la categoría de gran fumador e inestables al presentar valores de intervalo de confianza muy amplios y en las categorías de fumador leve a moderado éstos fueran no significativos. Así también, se piensa que en los controles puede haber sesgo de Berkson debido a que las prevalencias de tabaquismo son mayores a las reportadas para la población general a nivel nacional, lo cual puede, al contrario de lo sucedido con el sesgo en los casos, disminuir los valores del riesgo.

El sesgo de Berkson se produce cuando el estudio se realiza con pacientes hospitalizados y son incorporados individuos con la patología en estudio como casos y se reclutan en el hospital en el momento que probablemente han sido hospitalizados por una condición ligada a la exposición a investigar, por lo que todos, o la gran mayoría de los casos, presentan la exposición en estudio, incrementando el valor del estimador ${ }^{38}$. Esta situación antes descrita es la que podría explicar los altos valores de riesgo en los grandes fumadores, dado que todos los casos fueron reclutados durante su estadía en el hospital.

En relación a los casos, también habría que considerar como posible limitación, que aquellos que fueron reclutados para el estudio, fueron aquellos que estaban en condiciones de responder la encuesta, por lo que su nivel de severidad era probablemente menor, al menos en el momento de la entrevista. Esto pudiera implicar eventualmente, que presenten una menor exposición al tabaquismo, al ser comparados con pacientes más graves, debido a que ha sido señalado que el mantener el consumo de tabaco es un fuerte predictor de sobrevida ${ }^{39}$, pero esta hipótesis no es comprobable en este estudio debido a 
que no se registró el grado de severidad del paciente.

Otra eventual limitación de este estudio es que los pacientes fueron reclutados sólo en Santiago y que los datos son extrapolados al resto de Chile. Estimamos que esta eventual limitación es más aparente que real, ya que en Santiago se concentra $40 \%$ de la población nacional, producto de la migración de habitantes desde otras regiones.

Entre las ventajas de este estudio se encuentra que es el primero a nivel nacional y que además considera la exposición a distintos contaminantes. Además se indagó la asociación con tabaquismo considerando distintas variables como exposición. En la diferencia por género, la asociación encontrada es similar a la que exhibían hace algunos años los países "más desarrollados", por lo que aparece como un desafío poder evaluar la evolución de esta asociación.

En relación al impacto epidemiológico, los valores encontrados son altos. Los estudios internacionales señalan valores de fracción etiológica similares a los estimados en este estudio. En un artículo se señalan valores cercanos a $70 \%$ de fracción atribuible poblacional en Vietnam $^{40} \mathrm{y}$

\section{REFERENCIAS}

1. Da Costa E Silva Vl, Koifman S. Smoking in Latin America: a major public health problem. Cad Saude Publica 1998; 3: 99-108.

2. Alvarado R, talavera G. La epidemia de tabaquismo en Chile: Evolución durante la última década. Rev Chil Salud Pública 2003; 2: 69-73.

3. Henley SJ, Thun MJ, Chao A, Calle EE. Association between exclusive pipe smoking and mortality from cancer and other diseases. J Natl Cancer Inst 2004; 11: 853-61.

4. Au JS, Mang OW, Foo W, Law SC. Time trends of lung cancer incidence by histologic types and smoking prevalence in Hong Kong 1983-2000. Lung Cancer 2004; 2: 143-52.

5. Sobue T, Suzuki T, Fujimoto I, Matsuda M, Doi O, Mori $\mathrm{T}$ ET AL. Case-control study for lung cancer and cigarette smoking in Osaka, Japan: Comparison with the results from western Europe. Jpn J Cancer Res 1994; 85: 464-73.

6. Dol R, Him AB, Kreyberg L. The significance of cell type in relation to the aetiology of lung cancer. $\mathrm{Br} \mathrm{J}$ Cancer 1957; 11: 43-8.

7. Machin D, CAmpbell MJ, Fayers PM, Pinol APY. Sample algo superior a $80 \%$ en India ${ }^{41}$. Cabe señalar que existe una diferenciación por sexo, en que las fracciones atribuibles son mayores en los hombres, situación que no ha sido comunicada.

Respecto de los indicadores de impacto como fracciones atribuibles, es necesario comentar que es necesario tener algunas consideraciones metodológicas en su estimación, debido a que pueden estar sub o sobreestimadas incluso después de haber sido ajustadas por variables confundentes, lo que estaría explicado por el efecto de confusión residual. Así mismo se ha señalado que la fuente de información de donde se obtengan los datos para realizar estos análisis también podría incidir en la precisión de su estimación ${ }^{42}$.

En esta investigación ha quedado de manifiesto, al igual que nos ha sucedido con el estudio similar realizado en enfermedad pulmonar obstructiva crónica ${ }^{43}$, que una gran proporción de los casos incidentes diagnosticados cada año en el país serían evitados si la población dejara de consumir tabaco, por esta razón es pertinente implementar y mantener los esfuerzos que se están realizando para disminuir el consumo de tabaco entre la población, especialmente los jóvenes.

Size Tables for Clinical Studies. Segunda edición. Blackwell Science. Oxford. New York. 1997.

8. LaChin JM. Power and Sample Size Evaluation for the McNemar Test With Application to Matched CaseControl Studies. Statistics in Medicine 1992; 11: 1239-51.

9. Lauritsen JM, Bruus M, MyatT MA. EpiData, version 2.1. An extended tool for validated entry and documentation of data. Odense, Denmark: The EpiData Association, 2001.

10. KuRITZ S, LANDIS JR. Attributable risk estimation from matched case-control data. Biometrics 1988; 44: 35567.

11. LEvin ML. The occurrence of lung cancer in man. Acta Unio int contra cancrum 1953; 9: 531-41.

12. SPSS para Windows. Versión 12.0.1. Chicago: SPSSInc, 2000.

13. STATACORP. Stata Statistical Software: Release 8.0. College Station, TX: Stata Corporation, 2003.

14. Jöckel KH, Ahrens W, Wichmann HE, Becher H, BolmAudorfF U, JahN I ET aL. Occupational and environmental hazards associated with lung cancer. Int J Epidemiol 1992; 21: 202-13.

15. Rusch HA, Howe GR, Jain M, Burch JD, Holowaty EJ, MiLER AB. Are female smokers at higher risk for 
lung cancer than male smokers? A case-control analysis by histologic type. Am J Epidemiol 1993; 138: 281-93.

16. Sankanarayanan $R$, Varghese $C$, Duffy SW, PadmakumaRY G, DAY NE, NAIR MK. A case-control study of diet and lung cancer in Kerala, South India. Int J Cancer 1994; 58: 644-9.

17. De Stefani E, Fierro L, Correa P, Fontham E, Ronco A, LARRINAGA M ET AL. Mate drinking and risk of lung cancer in males: A case-control study from Uruguay. Cancer Epidemiol Biomarkers Prev 1996; 5: 515-9.

18. De Stefani E, Fierro L, Correa P, Fontham E, Ronco A, LARRINAGA M ET AL. Mate drinking and risk of lung cancer in males: A case-control study from Uruguay. Cancer Epidemiol Biomarkers Prev 1996; 5: 515-9.

19. Pawlega J, Rachtan J, Dyba T. Evaluation of certain risk factors for lung cancer in Cracow (Poland)-A case control study. Acta oncol 1997; 36: 471-6.

20. Schwartz AG, Swanson GM. Lung cancer in African American and whites. A population based study in metropolitan Detroit, Michigan. Cancer 1997; 79: 4552.

21. Jöckel KH, Ahrens W, Jahn I, Pohlabeln H, BolmAUDORFF U. Occupational risk factors for lung cancer: A case control study in West Germany. Int J Epidemiol 1998; 27: 549-60.

22. Wunsch-Filho V, Moncau JE, Mirabeli D, Boffetta P. Occupational risk factors of lung cancer in Sao Paulo, Brazil. Scand J Work Environ Health 1998; 24: 118-24.

23. Osann KE, Lowery JT, Schell MJ. Small cell lung cancer in women: Risk associated with smoking, prior respiratory disease and occupation. Lung cancer 2000; 28: 1-10.

24. Mao Y, Hu J, Ugnat AM, Semenciw R, Fincham S. Socioeconomic status and lung cancer risk in Canada. Int J Epidemiol 2001; 30: 809-17.

25. Bhurgi Y, Deculuer E, Bhurgi A, Nassar S, Usman A, BRENNAN P ET AL. A case-control study of lung cancer in Karachi, Pakistan. Int J Cancer 2002; 98: 952-5.

26. OsanN KE. Lung cancer in women: The importance of smoking, family history of cancer and medical history of respiratory disease. Cancer Res 1991; 54: 44-8.

27. Pezzotto SM, Mahuad R, Bay ML, Morini JC, Poletto L. Variation in smoking related lung cancer risk factors by cell type among men in Argentina: A case control study. Cancer causes control 1993; 4: 231-7.

28. Benhamou S, Benhamou E, Auquier A, Flamant R. Differential effects of tar content by type of tobacco and use of a filter on lung cancer risk in male cigarette smokers. Int J Epidemiol 1994; 23: 437- 43.
29. Suzuki I, Hamada GS, Zamboni MM, Cordeiro PD, Watanabe S, Tsugane S. Risk factors for lung câncer in Rio de Janeiro, Brazil: A case-control study. Lung cancer 1994; 11: 179-90.

30. RAchtAN J, SoKoLowski A. Risk factors for lung cancer among women in Poland. Lung cancer 1997; 35: 129-36.

31. Wakai K, Ohno Y, Genka K, Ohmine K, KaWamura T, TAMAKoshi A ET AL. Smoking habits, local brand cigarettes and lung cancer risk in Okinawa, Japan. J Epidemiol 1997; 7: 99-105.

32. Khuder SA, Dayal HH, Mutgi aB, Wiley JC, Dayal G. Effect of cigarette smoking on major histological types of lung cancer in men. Lung cancer 1998; 22: 15-21.

33. Agudo A, Ahrens W, Benhamou E, Benhamou S, BofFetTTA P, DARBy SC ET AL. Lung cancer and cigarette smoking in women: A multicenter case control study in Europe. Int J cancer 2000; 88: 820-7.

34. RACHTAN J. Smoking, passive smoking and lung cancer cell types among women in Poland. Lung cancer 2002: 35: 129-36.

35. Patel J, Bach P, KRIS M. Lung cancer in US women. A contemporary epidemic. JAMA 2004; 291: 1763-8.

36. Chile. Ministerio de Salud. Encuesta Nacional de Salud 2003. Disponible en: http://www.minsal.cl [consultado 24 agosto de 2004].

37. Quoix E, MeNNECIER B. What's new in the epidemiology of lung cancer: the female aspect. Breathe 2006; 2: $339-44$

38. Flanders WD, Boyle CA, Boring JR. Bias associated with differential hospitalization rates in incident casecontrol studies. J Clin Epidemiol 1989; 42: 395-401.

39. Nordquist L, Simon G, Cantor A, Alberts M, Bepler G. Improved survival in never smokers vs current smokers with primary adenocarcinoma of the lung. Chest 2004; 126: 347-51.

40. NGOAN LE T. Anti-smoking initiative and decline in incidence rates of lung cancer in Viet Nam. Asian Pac J Cancer Prev 2006; 7: 492-4.

41. Dikshit RP, Kanhere S. Tobacco habits and risk of lung, oropharyngeal and oral cavity cancer: a population-based case-control study in Bhopal, India. Int J Epidemiol 2000; 29: 609-14.

42. Malarcher AM, Schulman J, Epstein L, Thun M, Mowery P, Pierce B ET AL. Methodological issues in estimating smoking-attributable mortality in the United States. Am J Epidemiol 2000; 6: 573-84.

43. Amigo H, Erazo M, Oyarzún M, Bello S, Peruga A. Tabaquismo y enfermedad pulmonar obstructiva crónica: Determinación de fracciones atribuibles. Rev Méd Chile 2006; 10: 1275-82. 